\title{
Intermittent Behavior of Local Electron Temperature in a Linear ECR Plasma*)
}

\author{
Shinji YOSHIMURA, Kenichiro TERASAKA ${ }^{1)}$, Eiki TANAKA ${ }^{1)}$, Mitsutoshi ARAMAKI ${ }^{2}$ \\ and Masayoshi Y. TANAKA ${ }^{1)}$ \\ National Institute for Fusion Science, 322-6 Oroshi, Toki 509-5292, Japan \\ ${ }^{1)}$ Interdisciplinary Graduate School of Engineering Science, Kyushu University, \\ 6-1 Kasuga-koen, Kasuga 816-8580, Japan \\ ${ }^{2)}$ College of Industrial Technology, Nihon University, 1-2-1 Izumi-cho, Narashino 275-8575, Japan
}

(Received 25 November 2014 / Accepted 17 February 2015)

\begin{abstract}
An intermittent behavior of local electron temperature has been observed in a linear laboratory plasma, where a high-temperature region that has circular cross-section is formed along the magnetic field line for a short period of time. The floating potential signals which contain sporadic large-amplitude negative spikes associated with the electron temperature intermittency are analyzed statistically for various gas discharges. All the probability density functions of waiting time for different gas discharges exhibit exponential distributions, indicating that the random nature of this phenomenon, or the underlying Poisson process, is a universal property. On the other hand, mean duration of the intermittent events depends on the atomic mass of discharge gas species, showing that the dynamics of heavier particles may play an important role in this intermittency.
\end{abstract}

(C) 2015 The Japan Society of Plasma Science and Nuclear Fusion Research

Keywords: intermittency, laboratory plasma, electron cyclotron resonance, local electron temperature, floating potential, probability density function, Poisson process

DOI: $10.1585 /$ pfr. 10.3401028

\section{Introduction}

Intermittency is one of the important topics in the field of plasma research. Intermittent transport of coherent filamentary structures called blobs has frequently been observed in the edge of many magnetically confined plasmas [1]. Because the convection of blobs significantly enhances cross-field transport and may leads to deterioration in edge plasma confinement, the statistical properties of fluctuations in the edge are crucially important. Intermittent transport of similar turbulent structures has also been studied by linear devices [2,3].

Recently, we have observed a new intermittent behavior of local electron temperature in a linear electron cyclotron resonance (ECR) plasma. In contrast to abovementioned studies in which bursty ion saturation current signals were observed, we have found sporadic largeamplitude negative spikes in floating potential signals measured by a Langmuir probe. Although similar floating potential fluctuations in a detached recombining plasma were reported by Ohno et al. [4], the change in floating potential was attributable to the space potential fluctuations. Whereas, in our case, the current-voltage characteristics obtained by the conditional averaging method has revealed that the negative spikes are caused by intermittent enhancement of electron temperature, $T_{\mathrm{e}}$ (More precisely, it should

author's e-mail: yshinji@nifs.ac.jp

*) This article is based on the presentation at the 24th International Toki Conference (ITC24). be said as an experimentally obtained effective electron temperature, since it has been evaluated by the slope of the probe characteristics taken by a cylindrical Langmuir probe). Moreover, the circular localization of high- $T_{\mathrm{e}}$ region on the plasma cross-section has also been revealed by a newly-developed High-impedance Wire Grid (HIWG) detector [5]. The diameter of the circular high- $T_{\mathrm{e}}$ region is about $30-40 \mathrm{~mm}$. This spatial feature has also been confirmed by ICCD imaging of spontaneous emission from the $\mathrm{Ne} 2 \mathrm{p}_{1}$ state at $585 \mathrm{~nm}$ [6].

In this paper, statistical properties of the floating potential $\left(V_{\mathrm{f}}\right)$ fluctuation associated with the local $T_{\mathrm{e}}$ imtermittency are investigated in terms of the probability density functions (PDFs) of waiting time and duration, especially focusing on their atomic mass dependence. This paper is organized as follows. The experimental setup and diagnostics are described in Sec. 2. Sec. 3 presents experimental results including the $V_{\mathrm{f}}$ time series measured in ECR discharges with different gas species and an example of ICCD imaging. In Sec. 4, we discuss the statistical properties of $V_{\mathrm{f}}$ time series and their atomic mass dependence. Finally, our conclusions are presented in Sec. 5.

\section{Experimental Setup}

The experiments were performed in the high density plasma experiment-I (HYPER-I) device [7, 8] at the National Institute for Fusion Science (NIFS). HYPER-I is a linear device that consists of a cylindrical vacuum cham- 
Table 1 Filling gas pressures and microwave powers for different gas discharges.

\begin{tabular}{lll} 
gas species & filling pressure & microwave power \\
\hline helium $(\mathrm{He})$ & 1.5 mTorr & $20 \mathrm{~kW}$ \\
neon $(\mathrm{Ne})$ & 1.0 mTorr & $10 \mathrm{~kW}$ \\
argon $(\mathrm{Ar})$ & 0.5 mTorr & $5 \mathrm{~kW}$ \\
xenon $(\mathrm{Xe})$ & $0.5 \mathrm{~m}$ Torr & $5 \mathrm{~kW}$ \\
\hline
\end{tabular}

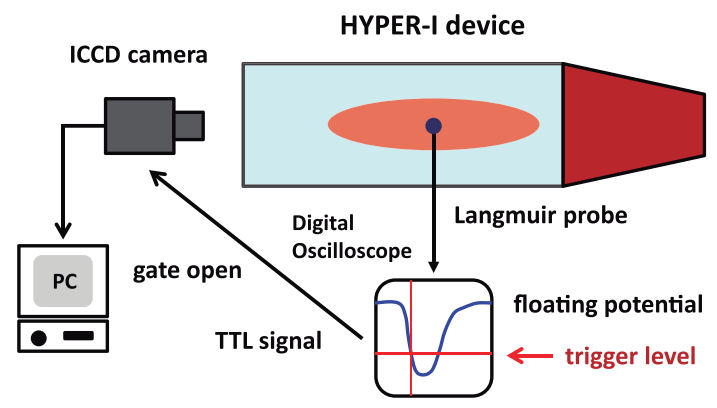

Fig. 1 Schematic of the experimental arrangement for ICCD imaging measurement.

ber, $0.3 \mathrm{~m}$ in diameter and $2.0 \mathrm{~m}$ in length, surrounded by a set of ten magnetic coils. The magnetic field configuration was a weakly-diverging one, where the ECR point for $2.45 \mathrm{GHz}$ microwave was located in the middle of the chamber in the axial direction. Plasmas were produced by ECR discharges of different gas species: helium, neon, argon, and xenon. The filling pressure and the microwave power used for each gas species are listed in Table 1. The discharge time was set to $20 \mathrm{~s}$ for $V_{\mathrm{f}}$ measurements.

The floating potential was measured with a cylindrical Langmuir probe terminated with a $1 \mathrm{M} \Omega$ resistor at $z=$ $1.175 \mathrm{~m}$, where $z$ stands for the axial distance measured from the microwave injection window. The size of the tungsten electrode tip was $1.5 \mathrm{~mm}$ in diameter and $1.0 \mathrm{~mm}$ in length. In order to ensure sufficient frequency response, a voltage follower circuit using a precision high-speed operational amplifier (OPA627, Burr-Brown) was directly connected to the probe [5]. The $V_{\mathrm{f}}$ signal was recorded with the DL750P ScopeCorder (Yokogawa), where the sampling rate was set to $2 \mathrm{MHz}$.

The spatial distribution of high- $T_{\mathrm{e}}$ region was measured by an intensified CCD camera (ICCD 576 MG/1, Princeton Instruments) with an interference filter for HeII emission at $468 \mathrm{~nm}$. Since the high- $T_{\mathrm{e}}$ region appears intermittently in time, the ICCD camera was operated by a trigger signal from $V_{\mathrm{f}}$ measurement with the Langmuir probe. The exposure time was set to $5 \mu \mathrm{s}$. Schematic of the experimental arrangement is shown in Fig. 1. Note that the obtainable image is a projection of line-integrated emission along the axis to the plasma cross-section, because the emission was measured through a viewing window installed at the end of the vacuum chamber.

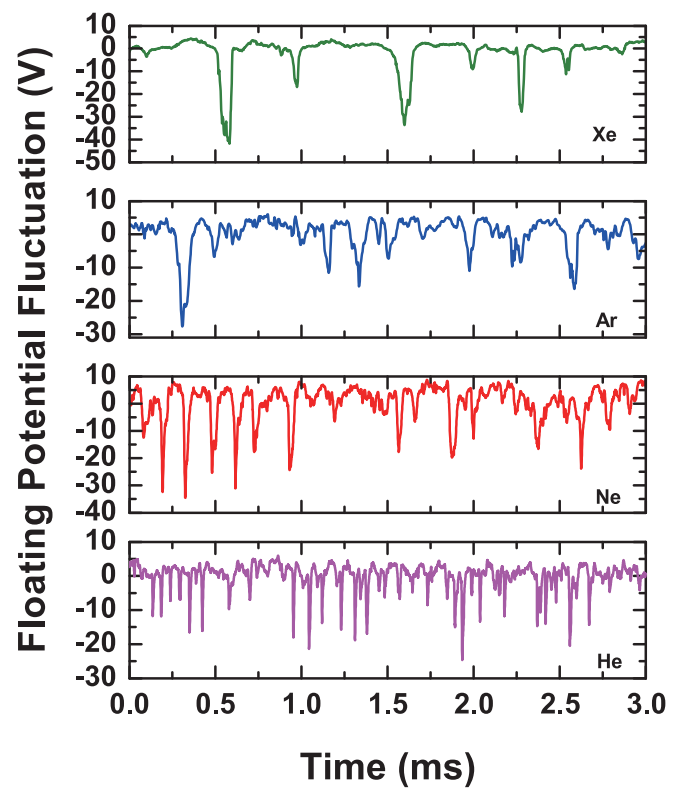

Fig. 2 Typical floating potential signals (fluctuating component) for different gas discharges. The gas species are helium, neon, argon, and xenon from bottom to top.

\section{Experimental Results}

\subsection{Negative spikes on floating potential signal}

Since floating potential $\left(V_{\mathrm{f}}\right)$ is, by definition, determined by the balance between influxes of electrons and ions, abrupt change in electron (ion) influx can give rise to a negative (positive) spike on the $V_{\mathrm{f}}$ signal. In general, $V_{\mathrm{f}}$ is a function of the space potential $\left(V_{\mathrm{s}}\right)$ and electron temperature $\left(T_{\mathrm{e}}\right)$. When $T_{\mathrm{e}}$ can be regarded as constant, $V_{\mathrm{f}}$ provides a good measure of $V_{\mathrm{s}}$ fluctuation. On the other hand, $V_{\mathrm{f}}$ can be used as an indicator of $T_{\mathrm{e}}$ fluctuation when $V_{\mathrm{s}}$ can be regarded as constant. The latter condition was satisfied in the present experiments, which was confirmed by the inflection point of conditionally averaged probe characteristics with and without the existence of the $V_{\mathrm{f}}$ spike.

Figure 2 shows typical fluctuating component of $V_{\mathrm{f}}$ extracted from full-length $(10 \mathrm{~s})$ time series, where the atomic mass of discharge gas species increases from bottom $(\mathrm{He})$ to top (Xe). The event magnitude in this study is defined by the peak amplitude of the negative spikes measured from the mean value. Therefore, the standard deviation $\sigma$ of full-length time series can be a good measure that describes how much the peak values deviate from the mean value. In the intense events, the amplitude of negative spikes attains more than $6 \sigma(\sigma \sim 3.8 \mathrm{~V}$ for He discharge), which can hardly be seen in usual Gaussian fluctuations. It is also evident that occurrence interval and duration of the negative spikes increase with increasing the atomic mass. The effects of atomic mass on the statistical properties of intermittent spikes are discussed in Sec. 4.

It should be pointed out that overall time-averaged plasma parameters are not affected by the presence of in- 


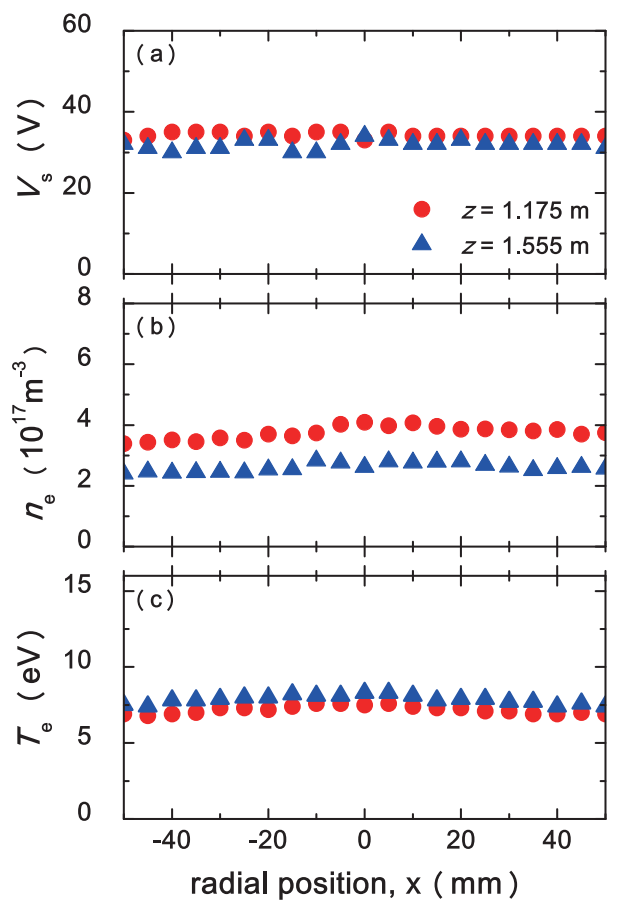

Fig. 3 Radial profiles of space potential, electron density, and electron temperature in a helium discharge. Circles and triangles stand for those quantities measured at $z=$ $1.175 \mathrm{~m}$ and $z=1.555 \mathrm{~m}$, respectively.

termittent spikes. Figure 3 shows radial profiles of space potential, electron density, and electron temperature in a helium discharge, which were obtained by conventional Langmuir probe measurement. All profiles are nearly uniform and have no steep gradient both in radial and axial directions. Therefore, spatial inhomogeneity is not the source of this intermittency.

\subsection{Spatial distribution of high $T_{\mathrm{e}}$ region}

Figure 4 shows an example of ICCD image of He-II line emission at $468 \mathrm{~nm}$. In order to obtain a clear image, 120 images were conditionally averaged for intense events in which the $V_{\mathrm{f}}$ fluctuation amplitude was larger than $47 \mathrm{~V}$ $(>12 \sigma)$. Circular bright region is clearly seen around the probe that was inserted to provide trigger signals for the ICCD camera. The diameter of the bright region is about $30 \mathrm{~mm}$, which is consistent with previous measurement using HIWG detector [5]. It should be emphasized that the occurrence positions of the bright region are not limited in the center of the plasma cross-section. The high- $T_{\mathrm{e}}$ region appears at various positions in a random manner. The position of the reference probe only determines the region of interest.

He-II line emission at $468 \mathrm{~nm}$ is not observed in usual HYPER-I plasmas, because the excitation energy for this transition is about $51 \mathrm{eV}$, which is much higher than typical bulk electron temperature $\left(T_{\mathrm{e}} \sim 10 \mathrm{eV}\right)$. In other words, it can be regarded as a good indicator of the high- $T_{\mathrm{e}}$ region.

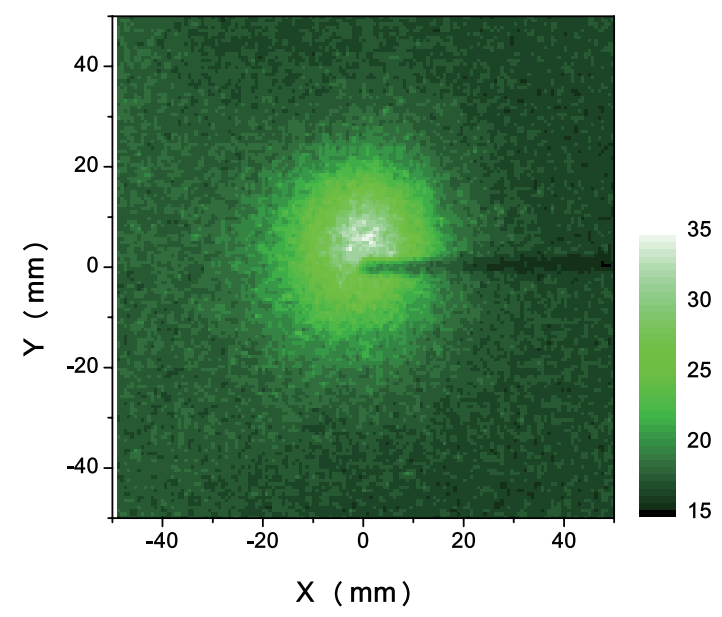

Fig. 4 An ICCD image of He-II line emission at $468 \mathrm{~nm}$.

Detailed spatio-temporal evolution of electron temperature in this intermittent phenomenon is beyond the scope of this paper and will be reported elsewhere.

\section{Discussions}

\subsection{Statistics of waiting time}

Here we have picked up the negative spikes of which amplitudes exceed $3 \sigma$ and defined them as the intermittent events of interest. In other words, the detection threshold value is three times the standard deviation. For example, the full-length time series of helium discharge contains more than 50,000 events by this definition.

In order to characterize such intermittent phenomenon, statistical analysis is a powerful tool. In particular, the statistics of waiting time [9] provides important information on mean occurrence rate and the randomness of the intermittent events. Here the waiting time is defined as the time interval between two minima of consecutive negative spikes whose amplitudes exceed $3 \sigma$.

Figure 5 shows the probability density functions (PDFs) of waiting time for different gas discharges in semilogarithmic plots. Each PDF is constructed from the corresponding 10-second time series presented in Sec. 3.1. In spite of apparent change in occurrence frequency in different gas discharges, all PDFs show obvious linear dependences. It has already been reported that the waiting time PDF of intermittent events for helium discharge obeys an exponential distribution [10]. It is remarkable that all the PDFs are well fitted with exponential distributions given by the following expression:

$$
P\left(\tau_{\mathrm{w}}\right)=\lambda \exp \left(-\lambda \tau_{\mathrm{w}}\right)
$$

where the waiting time $\left(\tau_{\mathrm{w}}\right)$ PDF is characterized by only one parameter $\lambda$.

This type of probability distribution for waiting time is naturally derived from a stationary Poisson process. The Poisson process is a random process in which all events occur independently with each other at a constant proba- 

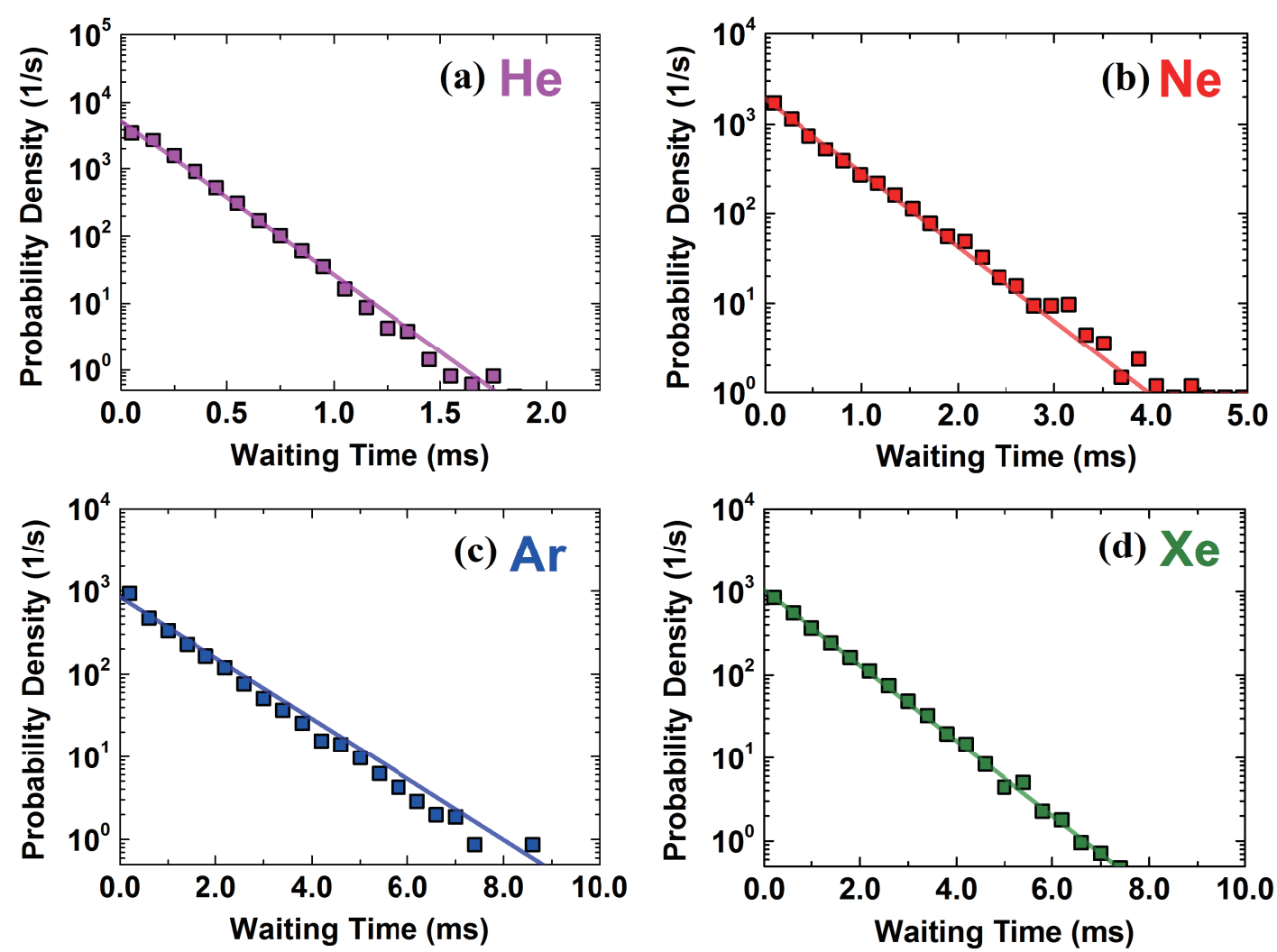

Fig. 5 Probability density functions of waiting time for (a) He, (b) Ne, (c) Ar, and (d) Xe discharges in semi-logarithmic scale. Solid lines are exponential distributions.

bility. The exponential distribution is a necessary condition for the Poisson process. A sufficient condition is that the probability for the number of events occurring in given time intervals is described by a Poisson distribution, which has also been confirmed in our previous work [10]. The exponential distribution of waiting time has also been found in blob transport in magnetically confined plasmas [11].

\subsection{Statistics of duration}

Duration time, which characterizes how long the event lasts, is another important parameter for spiky events. In this study, the duration is defined by the period for successive crossing of the threshold value.

Figure 6 shows the PDFs of duration for different gas discharges in logarithmic scale. A power-law dependence $\left(\propto \tau^{-5}\right)$ can be seen in the longer duration side of the PDFs; however, the origins of such dependence is still an open question. Another important property of the duration PDF seen in Fig. 6 is that the mean value of each gas discharge increases with increasing the atomic mass.

In order to investigate the atomic mass dependence, the mean durations are plotted as a function of atomic mass, which is shown in Fig. 7. A clear dependence that the mean duration is proportional to the square root of the atomic mass can be seen, which indicates a possible important role of heavier particles in the local electron temperature intermittency in linear ECR plasmas. It should be em-

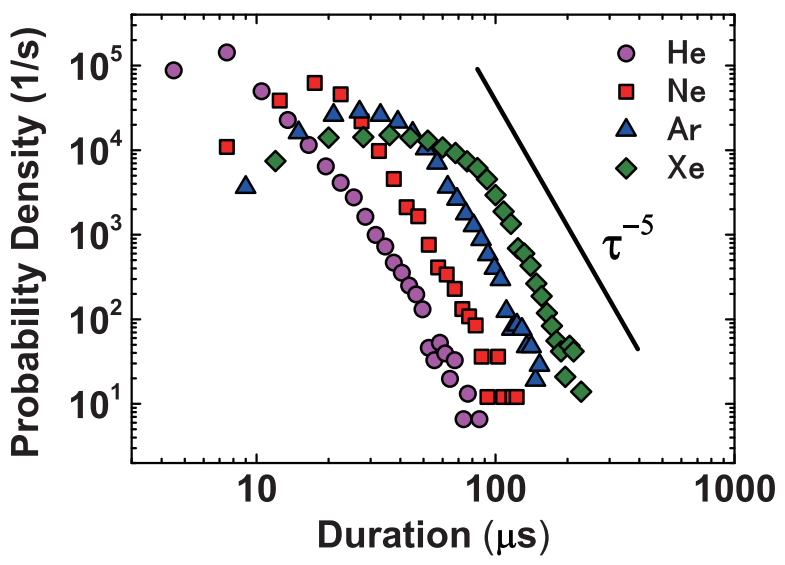

Fig. 6 Probability density functions of duration for different gas discharges in logarithmic scale.

phasized that the electron temperature intermittency presented in this article is not understandable by wave-particle interaction (electron cyclotron resonance) only. Detailed measurement of the dynamics of heavier particle is our future work.

\section{Conclusions}

Intermittent behavior of local electron temperature has been investigated in a linear ECR plasma with various gas 


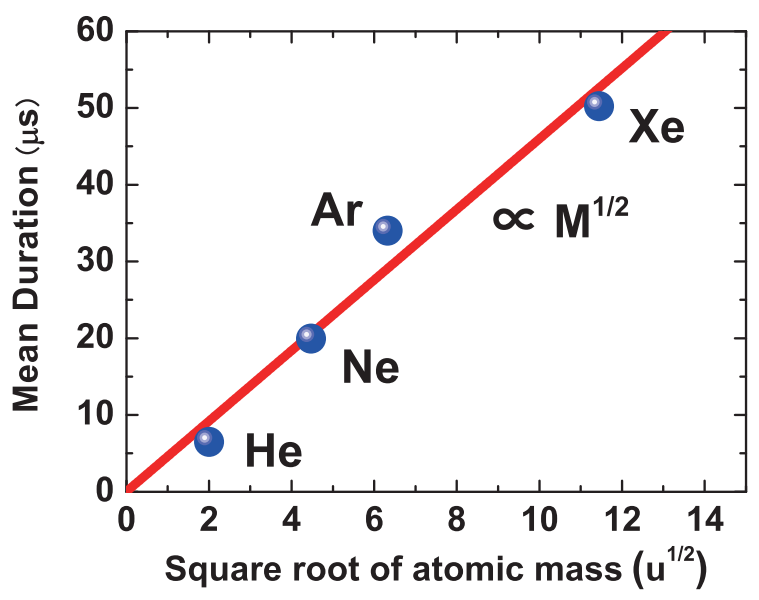

Fig. 7 Mean duration of the intermittent events for different gas discharge as a function of the square root of atomic mass.

species. The circular localization of high electron temperature region has been confirmed by ICCD imaging of He-II line emission at $468 \mathrm{~nm}$. Statistical analysis of the floating potential time series has revealed that the phenomenon is characterized by a stationary Poisson process of which probability density function of waiting time is given by an exponential distribution. The mean duration time of the intermittent events is proportional to the square root of the atomic mass of discharge gas, which suggests that the dynamics of heavier particle may play an important role in this electron temperature intermittency.

\section{Acknowledgments}

The authors thank Prof. T. Morisaki for having fruitful discussions on two-dimensional measurement of the intermittent phenomenon. This work was supported by JSPS KAKENHI Grant Number 24540554 and by NIFS Collaboration Research Program NIFS13KBAP016.

[1] G.S. Xu et al., Nucl. Fusion 49, 092002 (2009).

[2] T.A. Carter, Phys. Plasmas 13, 010701 (2006).

[3] T. Windisch et al., Plasma Phys. Control. Fusion 53, 085001 (2011).

[4] N. Ohno et al., J. Plasma Fusion Res. 78, 1093 (2002) [in Japanese].

[5] K. Terasaka et al., Rev. Sci. Instrum. 85, 113503 (2014).

[6] S. Yoshimura et al., IEEE Trans. Plasma Sci. 42, 2554 (2014).

[7] M.Y. Tanaka et al., Rev. Sci. Instrum. 69, 980 (1998).

[8] S. Yoshimura et al., to be published in J. Plasma Phys. 81 (2015).

[9] M. Aschwanden, Self-Organized Criticality in Astrophysics (Springer, Berlin, 2011) Chap.5.

[10] S. Yoshimura et al., JPS Conf. Proc. 1, 015030 (2014).

[11] S. Banerjee et al., Nucl. Fusion 52, 123016 (2012). 